\title{
Agent Optimization: Absorption, Distribution, Metabolism, Excretion, Dose, and Decay
}

\author{
Martin W. Brechbiel
}

Radiation Oncology Branch, National Cancer Institute, NIH (NCI Scientist Emeritus), Bethesda, Maryland

$\mathbf{T}$ argeted radiation therapy (TRT) is undergoing another renaissance attributable to U.S. Food and Drug Administration approvals of ${ }^{223} \mathrm{RaCl}_{2}$ (Xofigo; Bayer) and ${ }^{177} \mathrm{Lu}$-DOTATATE (Lutathera; Advanced Accelerator Applications) in 2013 and 2018, respectively. The former, though not a TRT agent, exploits nature's preferences for its biologic deposition. Its approval opened an acceptability window to use $\alpha$-emitting radionuclides in a clinical application. The latter combines ${ }^{177} \mathrm{Lu}\left(\beta^{-}\right.$-emitter) with the somatostatin analog DOTATATE, resulting in a peptide receptor radionuclide therapy TRT agent. This progress since the approval of ${ }^{90}$ Y-ibritumomab tiuxetan (Zevalin; IDEC) in 2002 is spurring continued optimism within this field. However, many fundamental paradigms and principles exist to reduce the period between renaissances (1).

A simple principle, often discounted, is balancing physical half-life of radionuclide with targeting-agent biologic half-life. Why is this important, and can it be achieved realistically? It is possible, if one accepts the physical limits imposed by choice of radionuclides. To maximize therapeutic impact, one aims to optimize delivery to and retention at the target to enhance actual radiation decay time on site. Unbound radionuclide is a potential source of toxicity until excreted and hence wasted. Mismatch of a short-half-life radionuclide with a slow-targeting, slowly cleared targeting agent or of a long-half-life radionuclide with a rapidtargeting, rapidly cleared targeting agent is suboptimal. Promising therapeutic results may occur, but careful optimization is important because significant improvement is almost certainly possible.

What is the best radionuclide? The answer depends on what is being treated, where it is located (accessibility), and what are the size and extent of disease. The entire list of radionuclides is largely irrelevant. Factoring in realistic half-lives (suitable for TRT), useful emissions (something cytotoxic), reasonably available production potential (can be made in useful quantity and purity), economic considerations (within funding confines), and meaningful chemistry (can be attached to targeting agents) reduces that list to about 20. Choices on emissions tend to be limited to $\beta^{-}$- and $\alpha$-emitters. Others are possible, such as Auger-emitters, but are pursued to a lesser degree (2). Most relevant research questions can be asked and answered with this short list.

The work of O'Donoghue et al. in 1995 very neatly provided a correlation between the ranges and energy depositions of $\beta^{-}$-emitters and the activity required for a cure probability of 0.9 , at an optimal tumor size (3). This ideal works well for patients presenting with

Received Nov. 12, 2020; revision accepted Nov. 19, 2020.

Published online Dec. 4, 2020.

COPYRIGHT (c) 2021 by the Society of Nuclear Medicine and Molecular Imaging. DOI: 10.2967/jnumed.120.258095 uniform tumors. It also provides a basis for the combination of distinct $\beta^{-}$-emitting radionuclides to cover a range of lesion sizes while balancing therapy with toxicity. It also provides the lower limit-that is, metastatic, single-cell, and small lesions-at which $\alpha$-emitters become effective.

The half-lives of the useful subset of radionuclides range from under an hour $\left({ }^{213} \mathrm{Bi}, 46 \mathrm{~min}\right)$ to weeks $\left({ }^{227} \mathrm{Th}, 18.7 \mathrm{~d}\right)(4)$. Fortunately, most radionuclides on that short list are reasonably available, albeit not at levels adequate to support commercialization. Past history ( ${ }^{90}$ Y-ibritumomab tiuxetan) does teach that Food and Drug Administration approval may significantly impact the production $(\uparrow)$ and cost $(\downarrow)$ of a radionuclide. Both ${ }^{211}$ At and ${ }^{149} \mathrm{~Tb}$ might be described as anchored to or limited in distribution from their production sites. The realities of shipping and distribution may limit use beyond a geographic range, despite demonstrations of efficacy, particularly when combined with limits in production technology. This may be preferable given the training requirement related to handling and administering these agents.

The radiolabeling chemistry for using most radionuclides on this short list exists, and much is commercially available. Some chemistry clearly would benefit from validated improvements in formation rates $\left({ }^{225} \mathrm{Ac}\right.$ and $\left.{ }^{227} \mathrm{Th}\right)$, and perhaps one will simply remain lacking $\left({ }^{223} \mathrm{Ra}\right)$. The real challenge here is in moving researchers toward validated chemistry, for example, use of an intact 1,4,7,10-tetraamino- $N, N^{\prime}, N^{\prime \prime}, N^{\prime \prime \prime}$ - tetracarboxylate cyclododecane instead of compromised derivatives, not reinventing wheels or using obsolete chemistry from dusty literature. This shift obviates continued waste of resources, compromised research, or studies that lack a true comparative nature facilitating clinical translation.

Economic considerations are straightforward. If a radionuclide cannot be made affordably, in suitable quantity and quality, then it is effectively unavailable. Fortunately, the field has access to a range of radionuclides of real potential clinical value.

The remaining challenge for TRT is in choosing the targeting agent. Criteria include highly selective and efficient targeting and binding, acceptable pharmacokinetics and dynamics, acceptable half-life and residence time (instant targeting, infinite retention on target, all excess instantly excreted), and realistic availability, production, and economics.

Pursuit of better targeting agents has delivered a broad range of options, yet their routine use has been limited. Full IgG antibodies and peptides were followed by $\mathrm{F}\left(\mathrm{ab}^{\prime}\right)_{2}$ and Fab fragments. These fragments filled gaps in the array of properties required to match radionuclide half-lives. Engineering of immunoproteins has effectively provided endless possibilities (5). Minibodies, flex minibodies, diabodies, and tetrabodies fill molecular size and associated targeting kinetics gaps. Remaining gaps down to the scale of peptides were filled with single-chain 
variable-fragment, single-domain antibody, and Affibody (Affibody $\mathrm{AB}$ ) formats. This matrix of agents provides serum half-lives from 1-3 wk to 30-60 min. Striking differences in biodistribution and tumor targeting are evident in comparing a ${ }^{177} \mathrm{Lu}-\mathrm{la}-$ beled IgG with a ${ }^{177}$ Lu-labeled single-domain antibody (6). Modification of minibody to flex-minibody further altered and increased tumor uptake and clearance kinetics (7). The impact by a diabody versus a minibody (and $\mathrm{sFv}-\mathrm{Fc}$ [single-chain variable fragment-fragment crystallizable]) on tumor residence time of $105 \mathrm{~h}$ versus $42 \mathrm{~h}$ is evident (8). The available diversity is well beyond the scope of this article. Yet, that same diversity of possible immunoprotein constructs illustrates the exquisite range of available targeting agents in the immunoprotein realm that should be exploited to optimize TRT.

Peptides, with a long history of modification to optimize target uptake, clearance route, and retention therein, clearly play a critical role as targeting agents. The impact of clearance route and retention is critical when developing therapeutics with a peptide-targeting agent because of molecular size. Dose incurred via renal excretion and retention from imaging agents may be significantly different when high-linear-energy-transfer or longer-range $\beta^{-}$-emissions are involved. Adjusting the functionality and substituents of an agent can clearly enhance renal excretion while reducing retention to facilitate the potential evolution of therapeutics (9). Pretargeting strategies can be viewed as a variant of peptide or small-molecule delivery platforms.

Once at the intersection of the matrix of radionuclides with potential targeting agent formats, one has to seriously question if the fundamental paradigm of matching radionuclide half-life to that of the targeting agent is fully addressed. Researchers should carefully choose radionuclides with optimal emissions related to penetration range and energies based on disease presentation. Next is to rationally match the half-life of the radionuclide to potential targeting agents. A mix-and-match, if/then, decision tree network could be envisioned. Defining appropriate leading candidates follows from performing the rote in vivo therapy experimentation studies with a full complement of controls to arrive at clinical trial candidates. Doing in vitro studies, cell killing, and similar studies has shown little usefulness as a predictor of therapeutic efficacy, particularly when crossing into the macromolecular in vivo (10). Empiric studies are inescapable. Information on radionuclides and targeting agents, the tools, has been readily available. Routine, systematic studies needed to generate and extract candidates for clinical translation are not generally being performed. Why might that be?

One could argue that the answer exists at the intersection of funding, concepts, and requirements governing academic research.
The outlined approach obviously requires funding. These studies are not overwhelmingly novel, can be both large and lengthy, may lack adequate stature to be published in highimpact journals, and may therefore be deemed unfundable by the peer-review process. As described, large empiric, rote in vivo therapy studies require significant investments in time and resources. Regardless, such studies are ultimately required to deliver optimal therapeutics. For similar reasons, critical subsequent toxicity studies are also largely unfundable and rarely publishable. Incremental peer-reviewed studies often fail to actually support clinical translation, despite appearing novel, whereas the lack of methodical and systematic studies to optimize matching radionuclide to targeting agent continues. Fundamental change in metrics for funding and publication of studies, such as weighting factors toward in vivo efficacy, is required. Shifting toward focused design and execution of comparative empiric studies, and broadening the aperture for funding and publishing beyond select institutions, will aid to keep this renaissance flourishing.

\section{DISCLOSURE}

No potential conflict of interest relevant to this article was reported.

\section{REFERENCES}

1. Boswell CA, Brechbiel MW. Development of radioimmunotherapeutic and diagnostic antibodies: an inside-out view. Nucl Med Biol. 2007;34:757-778.

2. Milenic DE, Brady ED, Brechbiel MW. Antibody targeted radiation cancer therapy. Nat Rev Drug Discov. 2004;3:488-499.

3. O'Donoghue JA, Bardiès M, Wheldon TE. Relationships between tumor size and curability for uniformly targeted therapy with beta-emitting radionuclides. $J$ Nucl Med. 1995;36:1902-1909.

4. Tsai WK, Wu AM. Aligning physics and physiology: engineering antibodies for radionuclide delivery. J Labelled Comp Radiopharm. 2018;61:693-714.

5. Freise $\mathrm{AC}, \mathrm{Wu} \mathrm{AM}$. In vivo imaging with antibodies and engineered fragments. Mol Immunol. 2015;67:142-152.

6. D'Huyvetter M, Vincke C, Xavier C, et al. Targeted radionuclide therapy with a ${ }^{177} \mathrm{Lu}$-labeled anti-HER2 nanobody. Theranostics. 2014;4:708-720.

7. Hu S, Shively L, Raubitschek A, et al. Minibody: a novel engineered anti-carcinoembryonic antigen antibody fragment (single-chain $\mathrm{Fv}-\mathrm{CH} 3$ ) which exhibits rapid, high-level targeting of xenografts. Cancer Res. 1996;56:3055-3061.

8. Williams LE, Wu AM, Kenanova VE, et al. Numerical comparison of iodinebased and indium-based antibody biodistributions. Cancer Biother Radiopharm. 2014;29:91-98.

9. Banerjee SR, Foss CA, Pullambhatla M, et al. Preclinical evaluation of ${ }^{86}$ Ylabeled inhibitors of prostate-specific membrane antigen for dosimetry estimates. J Nucl Med. 2015;56:628-634.

10. Duncan R. Drug-polymer conjugates: potential for improved chemotherapy. Anticancer Drugs. 1992;3:175-210. 